\title{
BMJ Open Interventions by healthcare professionals to improve management of physical long-term conditions in adults who are homeless: a systematic review protocol
}

\author{
Peter Hanlon, ${ }^{1}$ Lynsey Yeoman, ${ }^{1}$ Regina Esiovwa, ${ }^{2}$ Lauren Gibson, ${ }^{2}$ \\ Andrea E Williamson, ${ }^{3}$ Frances S Mair, ${ }^{1}$ Richard Lowrie ${ }^{2}$
}

To cite: Hanlon $\mathrm{P}$, Yeoman $\mathrm{L}$, Esiovwa R, et al. Interventions by healthcare professionals to improve management of physical long-term conditions in adults who are homeless: a systematic review protocol. BMJ Open 2017;7:e016756. doi:10.1136/ bmjopen-2017-016756

- Prepublication history and additional material for this paper are available online. To view these files please visit the journal online (http://dx.doi. org/10.1136/bmjopen-2017016756).

Received 8 March 2017 Revised 27 June 2017 Accepted 28 June 2017

\section{CrossMark}

${ }^{1}$ General Practice and Primary Care, Institute of Health and Wellbeing, University of Glasgow, Glassgow, UK ${ }^{2}$ Pharmacy Prescribing and Support Unit, West Glasgow Ambulatory Care Centre, NHS Greater Glasgow and Clyde, Glasgow, UK

${ }^{3}$ General Practice and Primary Care, School of Medicine, Dentistry and Nursing, University of Glasgow, Glassgow, UK

Correspondence to Dr Richard Lowrie; Richard.Lowrie@ggc.scot. nhs.uk

\section{ABSTRACT}

Introduction People experiencing homelessness are at increased risk of, and have poorer outcomes from, a range of physical long-term conditions (LTCS). It is increasingly recognised that interventions targeting people who are homeless should be tailored to the specific needs of this population. This systematic review aims to identify, describe and appraise trials of interventions that aim to manage physical LTCs in homeless adults and are delivered by healthcare professionals.

Methods and analysis Seven electronic databases (Medline, EMBASE, Cochrane Central Register of Controlled Trials, Assia, Scopus, PsycINF0 and CINAHL) will be searched from 1960 (or inception) to October 2016 and supplemented by forward citation searching, handsearching of reference lists and searching grey literature. Two reviewers will independently review titles, abstract and full-texts using DistillerSR software. Inclusion criteria include (1) homeless adults with any physical LTC, (2) interventions delivered by a healthcare professional (any professional trained to provide any form of healthcare, but excluding social workers and professionals without health-related training), (3) comparison with usual care or an alternative intervention, (4) report outcomes such as healthcare usage, physical and psychological health or well-being or cost-effectiveness, (5) randomised controlled trials, non-randomised controlled trials, controlled beforeafter studies. Quality will be assessed using the Cochrane EPOC Risk of Bias Tool. A meta-analysis will be performed if sufficient data are identified; however, we anticipate a narrative synthesis will be performed.

Ethics and dissemination This review will synthesise existing evidence for interventions delivered by healthcare professionals to manage physical LTCs in adults who are homeless. The findings will inform the development of future interventions and research aiming to improve the management of LTCs for people experiencing homelessness. Ethical approval will not be required for this systematic review as it does not contain individual patient data. We will disseminate the results of this systematic review via conference presentations, healthcare professional networks, social media and peer-reviewed publication.

\section{Strengths and limitations of this study}

- This systematic review will synthesise the evidence for interventions to manage physical long-term conditions in adults who are homeless.

- We expect this review will make a substantive contribution to the literature on how to improve health outcomes for this marginalised patient group.

- We anticipate significant clinical and methodological heterogeneity in eligible studies and expect to undertake a narrative synthesis.

Trial registration number PROSPERO registration number: CRD42016046183.

\section{INTRODUCTION}

The experience of homelessness is a dynamic, complex and multifaceted state, associated with increased morbidity ${ }^{1-4}$ and mortality. ${ }^{5}$ The prevalence of homelessness is increasing across high-income countries. ${ }^{7-10}$ There is considerable overlap between the experience of homelessness and other forms of 'deep social exclusion' such as previous experience of institutional care, substance misuse and 'street-culture activities' such as begging or 'survival' sex work. ${ }^{11}$ Multiple exclusion homelessness (MEH) has been used to refer to individuals experiencing homelessness plus any other deep social exclusion. Socioeconomic deprivation in general, ${ }^{12}{ }^{13}$ as well as specific features of adversity over the life course, ${ }^{14}$ are important risk factors for physical long-term conditions (LTCs). The overlap of various social exclusions in addition to risk factors for physical ill health has important implications for the type of services and the mode of engagement and delivery that is 
likely to be of benefit to people experiencing homelessness. ${ }^{1516}$

\section{Burden of physical LTCs}

Some physical LTCs, such as early-onset cardiac disease, ${ }^{17}$ chronic obstructive pulmonary disease,${ }^{18}$ some cancers, ${ }^{19}$ $\mathrm{HIV}^{20}$ and hepatitis $\mathrm{C},{ }^{21}$ are more prevalent in homeless populations than the general population. Other conditions, such as diabetes and (in some studies) hypertension, have a similar prevalence in homeless and non-homeless populations. ${ }^{22-24}$ All of these conditions, however, are more poorly controlled and have higher rates of complications in people who experience homelessness. ${ }^{1725}$ Adherence to treatment and engagement with services is also typically lower due to a combination of barriers relating to access, transportation and conflicting priorities. ${ }^{26-28}$ People experiencing homelessness may delay presenting to health services for preventative care, appearing only when sick or injured or when in need of medicines for pain or mental distress. ${ }^{29}$ Many people who are homeless have multiple physical and mental health problems and experience multimorbidity earlier and with greater severity. ${ }^{30}$ Such multimorbidity impacts negatively on functional status. Healthcare costs are also increased, in part through poorly coordinated use of health services. ${ }^{30}$ Rates of hospital admission and emergency department attendance are higher among people experiencing homelessness, ${ }^{31-34}$ although the latter is largely accounted for by markedly increased use in a small proportion of the homeless population. ${ }^{35}{ }^{36}$ Hospital stays are, on average, longer and associated with increased costs compared with those of housed patients. ${ }^{3738}$ Age-related functional decline ${ }^{39}{ }^{40}$ as well as hospital admission for a range of medical problems, ${ }^{41}$ tend to occur earlier in life among homeless people.

\section{Rationale for review}

Despite homelessness rates increasing, and being associated with a greater prevalence of physical and mental morbidity impacting negatively on healthcare costs and mortality, there remains a lack of robust evidence of effectiveness and cost-effectiveness of interventions, delivered by healthcare professionals, to improve health outcomes of people who are homeless. ${ }^{3042} 43$ This has led to calls for further research aiming to generate evidence of effective and cost-effective models of care, particularly for those in the extreme margins of social disadvantage, for example, rough sleepers, where the most profound evidence gap exists. ${ }^{44}$ Models for the delivery of healthcare to people experiencing homelessness include specialised primary care systems and integrated housing and health interventions; however, controlled evaluations of such models are relatively few and optimal delivery is likely to vary between different health and social care systems. ${ }^{45-49}$

Previous reviews have identified the potential benefit of interventions and strategies for mental health and substance misuse that are specifically tailored for and targeted at homeless people. ${ }^{46} 47$ Hwang et al identified better health outcomes following coordinated treatment programmes for homeless adults with mental illness or substance abuse or monetary incentives for homeless people with latent tuberculosis. ${ }^{46}$ Fitzpatrick-Lewis et al explored the effect of health and/or housing interventions on housing status. They found that abstinent-dependent housing improved outcomes more than non-abstinence dependent or no housing. These outcomes included increased housing tenure, increased substance abstinence and improved psychiatric outcomes. Provision of housing also improved health outcomes among homeless populations with HIV. ${ }^{47}$ Evaluations of a number of interventions targeting the physical health of people experiencing homelessness have been published since these systematic reviews. ${ }^{50}{ }^{51}$ However, to the authors' knowledge, no previous systematic reviews have focused specifically on the management of physical LTCs for people who are homeless. The increased prevalence of some physical LTCs, generally poorer LTC outcomes, higher complication rates and well-documented barriers to healthcare in homeless populations suggests that an evaluation of interventions focused specifically on physical LTC management in homeless people would be of value to service providers and to inform subsequent research. This paper describes the protocol for a systematic review to examine interventions by healthcare professionals targeting the management of physical LTCs among homeless and marginally housed adults.

\section{Aims}

This review aims to systematically identify, describe and appraise trials of interventions focusing on the management of physical LTCs, delivered by healthcare professionals to homeless adults. The review sets out to answer the following questions:

1. What are the key components of interventions aimed at optimising physical LTC management in homeless adults including theoretical underpinnings?

2. What outcome measures have been used in trials of interventions aimed at optimising physical LTC management in homeless adults and what effects, if any, have been reported?

\section{METHODS}

This systematic review protocol is registered with PROSPERO (registered on 02/12/2016, PROSPERO ID: CRD42016046183, available from http://www. crd.york.ac.uk/PROSPERO/display_record.asp? ID= CRD42016046183).

\section{Criteria for eligibility for inclusion}

Inclusion criteria for this systematic review are presented according to PICOS (Population, Intervention, Comparator, Outcomes, Study design) criteria. These are summarised in table 1 . 
Table 1 PICOS inclusion criteria

\section{PICOS component Description}

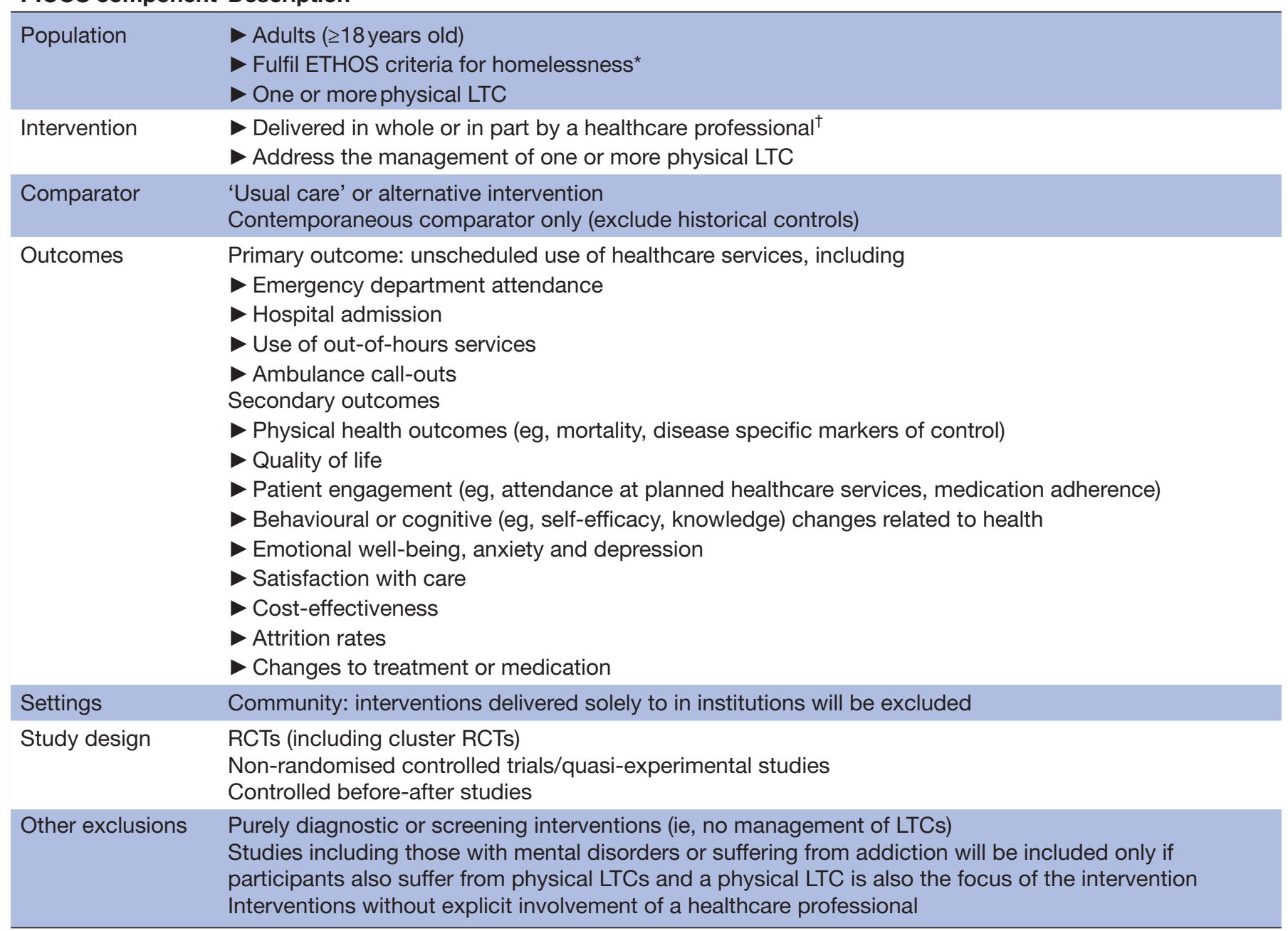

*See 'Population' section in text.

†Including, but not limited to, physicians, nurses, dentists, pharmacists, paramedics, mental health professionals, allied health professionals (eg, physiotherapists, dieticians, clinical psychologists, etc), midwives.

ETHOS, European Typology of Homelessness and Housing Exclusion; LTC, long-term condition; PICOS, Population, Intervention,

Comparator, Outcomes, Study design; RCT, randomised controlled trial.

\section{Population}

Various definitions of homelessness have been developed, often to define eligibility for access to services; however, no internationally agreed definition exists. In general, in addition to 'rough-sleeping' individuals, definitions also include those in insecure or inadequate housing, those without a permanent abode and those at risk of eviction. The European Federation of National Organisations Working with the Homeless developed a European Typology of Homelessness and Housing Exclusion (ETHOS), which considers physical, social and legal domains of having a home, deficits in which can be seen as leading to the four main categories of homelessness described in the typology. ${ }^{52}$ These comprise:

- Rooflessness-those without shelter

- Houselessness-those in temporary or institutional accommodation
- Insecurely housed-those in insecure accommodation or at risk of eviction

- Inadequately housed-including unfit housing, extreme overcrowding or temporary non-conventional structures

For the purposes of this review, we will adopt the ETHOS definition. Studies including a broader population but including homeless participants will be included only if data pertaining to homeless participants are considered separately.

For the purposes of this review, we will include only physical LTCs and exclude mental health and substance misuse. This decision was made as existing systematic reviews of healthcare interventions for homeless people have synthesised evidence on mental health and substance misuse interventions. ${ }^{46} 47$ Studies including those with mental disorders or substance misuse will be included 
only if participants also suffer from physical LTCs, and a physical LTC is also the focus of the intervention. We will include studies of homeless individuals with a specific physical LTC (eg, those with HIV, diabetes etc) or those which include those with a range of physical conditions.

\section{Intervention}

Studies will be included if they concern non-pharmacological interventions, delivered (in whole or in part) by a healthcare professional, addressing the management of one or more physical LTC. We will exclude studies evaluating only a specific drug or pharmacological agent. In contrast, we will include studies that have such interventions (the prescription of a specific drug) as a component so long as the intervention also involves a broader healthcare professional intervention such as a change in role or training for a healthcare professional or a change in the organisation or delivery of care.

Many LTCs may be under-recognised or undiagnosed in homeless individuals. ${ }^{26}$ As such we will include interventions which involve screening for or diagnosis of physical LTCs if the subsequent management of the condition is also part of the intervention. Solely screening or diagnostic interventions will be excluded.

\section{Outcomes}

Our primary outcomes are measures of unscheduled healthcare usage. Given the potentially wide range of LTCs eligible for inclusion in the review, we do not propose to provide an exhaustive list of these, but will extract and report such outcomes as they are described in eligible studies. Examples may include emergency department attendance, hospital admission (either all-cause of disease specific), use of out-of-hours services and so on. Secondary outcomes of mortality and markers of disease control will also be included however reported, whether general or disease specific (eg, viral load in hepatitis C or haemoglobin A1c in diabetes etc). Studies would be eligible for inclusion if they report any of the primary or secondary outcomes.

\section{Setting}

Trials would be eligible for inclusion if they focus on the management/support of homeless individuals in the community. Interventions that are initiated as an inpatient, but continue in the community, would be eligible for inclusion, but interventions that are solely undertaken in an inpatient setting will be excluded. Interventions delivered solely to institutionalised individuals or inpatients will be excluded.

\section{Identification of studies}

\section{Electronic searches}

The following databases will be searched using a combination of keywords and MeSH terms: Medline, Embase, Cochrane Central Register of Controlled Trials , Assia, Scopus, PsycINFO and CINAHL. A full list of search terms for Medline is shown in box and will be adapted for other databases. Searches will be from 1960 to October

\section{Box Medline search strategy*}

1. Exp. Homeless Persons/

2. Home?less.mp

3. Roof?less.mp

4. House?less.mp

5. (home ${ }^{\star}$ adj2 lack).mp

6. (home* adj2 no).mp

7. (without adj2. Home*).mp

8. (lack adj2 hous $\left.{ }^{\star}\right) \cdot \mathrm{mp}$

9. (no adj2 hous*).mp

10. (without adj2. hous*).mp

11. (lack adj2 roof*).mp

12. (no adj2 roof*).mp

13. (without adj2 roof*).mp

14. (inadequate* adj3 hous*).mp

15. (insecur* adj3 hous $\left.{ }^{\star}\right) \cdot \mathrm{mp}$

16. (insecur* adj2 tenan*).mp

17. (unfit* adj2 hous*).mp

18. ((transition* or insecure or inadequate or substandard or substandard or sheltered or emergency or intermittent or transient or marginal* or problem*) adj (hous* or home* or accommodat*)).mp

19. (sheltered or unsheltered or shelters).mp

20. Vagran*.mp

21. Destitute.mp

22. Skid row.mp

23. (sleep* adj2 rough).mp

24. ("street person" or "street people"). Mp

25. Exp "Delivery of Health Care"/

26. Exp Primary Health Care/

27. Exp Community Health Services/

28. Exp Chronic Disease

29. ((chronic or long term) adj2 (disease or condition*)).mp

30. Exp Patient Care Management/

31. Intervention*.mp

32. Exp Pragmatic Clinical Trial/ or exp Clinical Trial/ or exp Randomized Controlled Trial/ or exp Controlled Clinical Trial/

33. Trial*.mp

34. Control*.mp

35. 1 or 2 or 3 or 4 or 5 or 6 or 7 or 8 or 9 or 10 or 11 or 12 or 13 or 14 or 15 or 16 or 17 or 18 or 19 or 20 or 21 or 22 or 23 or 24

36. 25 or 26 or 27 or 28 or 29 or 30

37. 31 or 32 or 33 or 34

38. 35 and 36 and 37

*Adapted for other databases.

2016. These will be supplemented by handsearching the Journal of the Poor and Underserved, handsearching reference lists of eligible studies and forward citation searches of included studies using Web of Science. No language restriction will be applied to the search, but only English language articles will be included at the screening level.

\section{Searching additional sources of literature}

We will also attempt to identify 'grey literature' by searching the following sources:

1. Websites of non-governmental organisations that aim to assist homeless persons. Search for reports, working papers, newsletters, protocols, conference papers/ 
posters and clinical trials that are relevant to the study. Organisations to be searched are KHP Pathway, Shelter Scotland, Crisis, Homeless link, Revolving doors agency, Lankelly Chase and the King's Fund.

2. Department of Health England webpage for relevant materials.

3. Google, Google scholar and Google Advanced.

4. OpenGrey, WorldCat, Grey Literature Report, OAlster and WorldWideScience for reports and theses.

5. British library and Zetoc for conference abstracts, reports and theses.

6. Research Councils UK information on publicly funded research.

7. Repositories including Grey Guide and Open DOAR.

8. Other related sites including UK health forum, St. Michael's hospital, Grey Net.

\section{Data collection and analysis}

Selection of studies

After removal of duplicates, all records identified through electronic searches will be uploaded onto 'DistillerSR' software. Titles and abstracts will be independently screened by two reviewers. Conflicts will be resolved by discussion, with studies retained for full-text screening if agreement is not reached. Where no abstract is available, records will be retained for full-text assessment unless they can be judged from the title not to relate to the review topic.

Full-texts of all potentially eligible studies will be obtained, uploaded onto DistillerSR and assessed for eligibility using a piloted checklist based on the above PICOS criteria. Conflicts will be resolved by discussion, involving a third reviewer where agreement cannot be reached. A list of all studies excluded at full-text level will be compiled along with reasons for exclusion.

\section{Data extraction}

For each study meeting the inclusion criteria, data will be extracted by two reviewers, working independently, using a piloted data extraction template (online supplementary file 1).

Bibliographic details to be extracted include author, country, year, journal/reference.

Participant characteristics will include: gender, age, ethnicity, definition of homelessness, details of comorbidity and method of recruitment. Methods of recruitment and retention will be extracted. Studies may recruit participants with a specific physical LTC or analyse LTCs in a broader group, in either case data on the LTC including disease severity, where available, will be collected. Details of medications will also be extracted.

Details of the intervention components will be extracted including data on: setting; mode of delivery; professional(s) involved; duration; frequency and intensity of each intervention. Reviewers will also characterise the components of the intervention using an adaptation of the EPOC taxonomy of health systems interventions. ${ }^{53}$ Any available detail of the control/comparator intervention will also be extracted, including any available detail of the components of 'usual care'. Information about theoretical underpinnings, if used, will also be included.

Results for primary and secondary outcomes (as described in 'Outcomes') will also be extracted using the piloted template. Due to the diverse range of potential secondary outcomes, all available outcome definitions from the included studies will be extracted along with the results to facilitate an assessment of the comparability of different studies. Dichotomous outcomes will be reported as proportions of participants or control in whom the outcome occurs, along with summary ORs and 95\% CIs for comparisons between groups. Continuous variables will be reported as mean difference between groups with 95\% CIs or, where available, mean change from baseline as well as mean difference in change between intervention and control groups with $95 \%$ CIs.

Where studies of relevant interventions are identified, but data relating to homeless participants or to LTCs are not reported separately, authors will be contacted to attempt to obtain and include these data.

\section{Assessment of methodological quality/risk of bias}

Risk of bias within each of the included studies will be assessed for each of the nine domains recommended for randomised controlled trials (RCTs), non-RCTs and controlled before-after studies in the Cochrane EPOC guidelines. ${ }^{53}$ Risk of bias relating to the primary outcome will be assessed and presented in a risk of bias table. We would plan to summarise the risk of bias for individual outcomes following assessment of included studies and synthesis of their findings.

\section{Data synthesis}

Results of the literature search, screening and reasons for inclusion and exclusion will be presented in a Preferred Reporting Items for Systematic Reviews and Meta-Analyses (PRISMA) diagram. ${ }^{54}$ Tables will be used to summarise the population, intervention, outcomes of interest and design of each included study. The risk of bias assessment of each study will also be presented as per the Cochrane EPOC guidelines. ${ }^{53}$

From our scoping of the literature, we expect to find considerable methodological and clinical heterogeneity. We anticipate, therefore, that a narrative synthesis will be most appropriate and will be conducted to describe the key characteristics, components, outcomes and underpinning theory of the interventions studied. Should several studies with sufficiently similar populations and outcomes be identified, we will assess statistical heterogeneity using an $\mathrm{I}^{2}$ statistic and combine results using a random effects meta-analysis only if appropriate. ${ }^{55}$

In conducting a narrative synthesis, should this be most appropriate, the findings from each of the included studies will be grouped by similar outcomes. For each study we will present the following information to provide a summary of the available data:

- The number of participants in the study. 
- Study design.

- The outcome-level risk of bias of the study.

- Findings for quantitative outcomes will be indicated as positive if statistically significant and favouring the intervention, negative if favouring the control or neutral if not statistically significant.

- Inconsistent findings within individual studies will be indicated.

- Attrition rates for included studies will be compared.

Two reviewers will perform all data extraction, outcome assessment and quality assessment independently. Any discrepancies or disagreements will be resolved by discussion, involving a third reviewer when necessary. Where studies consider similar outcomes, but a meta-analysis is not appropriate due to heterogeneity, we will calculate and compare effect sizes for these outcomes. Important methodological differences will be highlighted in the review text. Finally a GRADE approach will be used to summarise findings for individual outcomes or groups of outcomes across included studies and indicate the strength of the available evidence. ${ }^{53}$

\section{DISCUSSION}

This systematic review will aim to synthesise the existing evidence for interventions delivered by healthcare professionals for physical LTC management with adult homeless people. We will aim to characterise and describe the interventions, including their individual components, before assessing their impact, if any, on healthcare usage, engagement with treatment and clinical outcomes. We will endeavour to analyse in detail not only the interventions themselves but the context, perceived difficulties, cost-effectiveness and level of participant engagement as reported so as to inform future intervention development and research in this sphere.

Innovations to health services and research into the best models of care are necessary to improve the health of people who are homeless. ${ }^{138}$ Primary healthcare for homeless persons in high-income countries commonly adopt a multidisciplinary approach. ${ }^{15657}$ Recent developments in professional roles, for example, prescribing pharmacists $;{ }^{58}$ shortages of traditional providers of primary care (general practitioners ${ }^{59}$ and nurses ${ }^{60}$ ) in the UK and increasing numbers of people who are homeless with associated multiple health and prescribing or medication-related problems provides a platform for diversification in primary healthcare delivery. ${ }^{61-63}$ This review is timely, as healthcare systems struggle to adapt and respond to the growing numbers of ageing people who are homeless and addresses an outstanding evidence gap.

It is likely, given our initial scoping of the topic area we will (as the results of previous systematic reviews of homeless health more generally) find that the quantity of available evidence will be limited. The study of homeless populations presents considerable challenges in conducting large-scale RCTs, in part due to the intrinsically transient nature of homelessness leading to engagement, participation, recruitment and retention difficulties. As a result we have chosen to include a range of study designs, while limiting to controlled studies as this was deemed crucial to assessing the efficacy of the interventions to be reviewed. The anticipated quantity of evidence as well as the inclusion of a range of study designs including some 'less-robust' designs is a potential weakness of the proposed review, but we would argue a necessary one to facilitate a meaningful assessment of the range of evidence available in an important area of study.

Given the aforementioned potential weakness, we recognise the importance of carefully describing and characterising the details of the interventions themselves as well as the limitations of the design of the individual studies. We will use and adapt a recognised taxonomy for health system intervention (EPOC taxonomy) as well as reporting a structured quality assessment to facilitate this. In view of the wide range of conditions and interventions which would be eligible for inclusion, leading to considerable potential heterogeneity, we anticipate that a narrative synthesis is likely to be the most valid method of analysis and presentation of findings. We expect such a synthesis will serve to inform clinicians and researchers in developing and delivering services to this marginalised patient group.

Acknowledgements We acknowledge the support of Catriona Deenoon, librarian for NHS Greater Glasgow and Clyde, for her support in advice in carrying out the scoping searches, designing the search strategy and piloting and finalising the search terms.

Contributors All authors (PH, LY, RE, LG, AEW, FSM and RL) contributed to the conception and design of the proposed study. PH, LY, RE, AEW, FSM and RL contributed to the development of data sources and search strategy. PH, LY, RE, AEW, FSM and RL developed and refined the inclusion criteria. PH, LY, RE, LG, FSM and $R L$ developed the data extraction template which was piloted by PH, LY and LG. $\mathrm{PH}$ wrote the first draft. All authors critically reviewed this and subsequent drafts of the manuscript and provided input into its content. All authors approved the final version of the manuscript to be published. $\mathrm{RL}$ is guarantor of the review. All authors accept accountability for the accuracy of the protocol.

Competing interests None declared.

Provenance and peer review Not commissioned; externally peer reviewed.

Data sharing statement Additional data, where applicable, are available on request from the corresponding author $\mathrm{RL}$.

Open Access This is an 0pen Access article distributed in accordance with the Creative Commons Attribution Non Commercial (CC BY-NC 4.0) license, which permits others to distribute, remix, adapt, build upon this work non-commercially, and license their derivative works on different terms, provided the original work is properly cited and the use is non-commercial. See: http://creativecommons.org/ licenses/by-nc/4.0/

(c) Article author(s) (or their employer(s) unless otherwise stated in the text of the article) 2017. All rights reserved. No commercial use is permitted unless otherwise expressly granted.

\section{REFERENCES}

1. Fazel S, Geddes JR, Kushel M. The health of homeless people in high-income countries: descriptive epidemiology, health consequences, and clinical and policy recommendations. Lancet 2014;384:1529-40. 
2. Lebrun-Harris LA, Baggett TP, Jenkins DM, et al. Health status and health care experiences among homeless patients in federally supported health centers: findings from the 2009 patient survey. Health Serv Res 2013;48:992-1017.

3. Schanzer B, Dominguez B, Shrout PE, et al. Homelessness, health status, and health care use. Am J Public Health 2007;97:464-9.

4. Sun S, Irestig R, Burström B, et al. Health-related quality of life (EQ5D) among homeless persons compared to a general population sample in Stockholm County, 2006. Scand J Public Health 2012;40:115-25.

5. Nusselder WJ, Slockers MT, Krol L, et al. Mortality and life expectancy in homeless men and women in Rotterdam: 2001-2010. PLoS One 2013;8:e73979.

6. Nielsen SF, Hjorthøj CR, Erlangsen A, et al. Psychiatric disorders and mortality among people in homeless shelters in Denmark: a nationwide register-based cohort study. Lancet 2011;377:2205-14.

7. United States Interagency Council on Homelessness. Opening doors: federal strategic plan to prevent and end homelessness. https://www.usich.gov/resources/uploads/asset_library/USICH OpeningDoors_Amendment2015_FINAL.pdf (accessed 24 Feb 2017).

8. United Kingdom Department for Communities and Local Government. 2013. Rough sleeping statistics England-August 2012 experimental statistics. London: Department for Communities and Local Government. https://www.gov.uk/government/collections/ homelessness-statistics. (accessed Feb 2017).

9. United Kingdom Department for Communities and Local Government. 2013. Statistical Data set. live tables on homelessness: statutory homelessness: households accepted by local authorities as owed a main homelessness duty, by ethnicity, England, 1998 to 2013. London: Department for Communities and Local Government. https://www.gov.uk/government/statistical-data-sets/live-tables-onhomelessness. (accessed 24 Feb 2017).

10. European Commission. Commission staff working document. confronting homelessness in the European Union. Brussels: European Commission, 2013. http://aei.pitt.edu/45917/1/swd2013 0042.pdf. (accessed $24 \mathrm{Feb} 2017$ ).

11. Fitzpatrick S, Johnsen S, White M. Multiple Exclusion homelessness in the UK: key patterns and intersections. Social Policy and Society 2011;10:501-12.

12. Dixon L, Postrado L, Delahanty J, et al. The association of medical comorbidity in schizophrenia with poor physical and mental health. $J$ Nerv Ment Dis 1999;187:496-502.

13. Barnett K, Mercer SW, Norbury M, et al. Epidemiology of multimorbidity and implications for health care, research, and medical education: a cross-sectional study. Lancet 2012;380:37-43.

14. Bellis MA, Hughes K, Leckenby N, et al. Measuring mortality and the burden of adult disease associated with adverse childhood experiences in England: a national survey. $J$ Public Health 2015;37:fdu065.

15. Hampson T. Hardest to reach? the politics of multiple needs and exclusions. London: Fabian Society, 2010. http://www.meam.org.uk/ wp-content/uploads/2010/04/Hardest-to-Reach.pdf. (accessed 24 Feb 2017).

16. Cornes M, Joly L, Manthorpe J, et al. Working together to Address Multiple Exclusion homelessness. Soc Policy Soc 2011;10:513-22.

17. Lee TC, Hanlon JG, Ben-David J, et al. Risk factors for cardiovascular disease in homeless adults. Circulation 2005;111:2629-35.

18. Snyder LD, Eisner MD. Obstructive lung disease among the urban homeless. Chest 2004;125:1719-25.

19. Baggett TP, Hwang SW, O'Connell JJ, et al. Mortality among homeless adults in Boston: shifts in causes of death over a 15-year period. JAMA Intern Med 2013;173:189-95.

20. Song JY, Safaeian M, Strathdee SA, et al. The prevalence of homelessness among injection drug users with and without HIV infection. J Urban Health 2000;77:678-87.

21. Beijer $U$, Wolf A, Fazel S. Prevalence of tuberculosis, hepatitis $C$ virus, and HIV in homeless people: a systematic review and metaanalysis. Lancet Infect Dis 2012;12:859-70.

22. Scott J, Gavin J, Egan AM, et al. The prevalence of diabetes, prediabetes and the metabolic syndrome in an irish regional homeless population. QJM 2013;106:547-53.

23. Oliveira LP, Pereira ML, Azevedo A, et al. Risk factors for cardiovascular disease among the homeless and in the general population of the city of Porto, Portugal. Cad Saude Publica 2012;28:1517-29.

24. Kubisová D, Adámková $\mathrm{V}$, Lánská $\mathrm{V}$, et al. Higher prevalence of smoking and lower BMI, waist circumference, cholesterol and triacylglyceride levels in Prague's homeless compared to a majority of the Czech population. BMC Public Health 2007;7:51.
25. Kim DH, Daskalakis C, Plumb JD, et al. Modifiable cardiovascular risk factors among individuals in low socioeconomic communities and homeless shelters. Fam Community Health 2008;31:269-80.

26. Argintaru N, Chambers C, Gogosis E, et al. A cross-sectional observational study of unmet health needs among homeless and vulnerably housed adults in three Canadian cities. BMC Public Health 2013;13:577.

27. Kushel MB, Vittinghoff E, Haas JS. Factors associated with the health care utilization of homeless persons. JAMA 2001;285:200-6.

28. Gelberg L, Andersen RM, Leake BD. The behavioral model for vulnerable populations: application to medical care use and outcomes for homeless people. Health Serv Res 2000;34:1273-302.

29. Elliott BA, Beattie MK, Kaitfors SE. Health needs of people living below poverty level. Fam Med 2001;33:361-6.

30. Brett T, Arnold-Reed DE, Troeung L, et al. Multimorbidity in a marginalised, street-health Australian population: a retrospective cohort study. BMJ Open 2014;4:e005461.

31. Bharel M, Lin WC, Zhang J, et al. Health care utilization patterns of homeless individuals in Boston: preparing for Medicaid expansion under the Affordable Care Act. Am J Public Health 2013;103 Suppl 2:S311-7.

32. Brown RT, Kiely DK, Bharel M, et al. Use of acute care services among older homeless adults. JAMA Intern Med 2013;173:1831-4.

33. Verlinde $\mathrm{E}$, Verdée $\mathrm{T}$, Van de Walle $\mathrm{M}$, et al. Unique health care utilization patterns in a homeless population in Ghent. BMC Health Serv Res 2010;10:242.

34. O'Carroll A, O'Reilly F. Health of the homeless in Dublin: has anything changed in the context of Ireland's economic boom? Eur J Public Health 2008;18:448-53.

35. Hwang SW, Chambers C, Chiu S, et al. A comprehensive assessment of health care utilization among homeless adults under a system of universal health insurance. Am J Public Health 2013;103 Suppl 2:S294-301.

36. Chambers $\mathrm{C}$, Chiu S, Katic M, et al. High utilizers of emergency health services in a population-based cohort of homeless adults. Am J Public Health 2013;103 Suppl 2:S302-S310.

37. Hwang SW, Weaver J, Aubry T, et al. Hospital costs and length of stay among homeless patients admitted to medical, surgical, and psychiatric services. Med Care 2011;49:350-4.

38. Hwang SW, Burns T. Health interventions for people who are Homeless. The Lancet 2014;384:1541-7.

39. Brown RT, Kiely DK, Bharel M, et al. Geriatric syndromes in older homeless adults. J Gen Intern Med 2012;27:16-22.

40. Garibaldi B, Conde-Martel A, O'Toole TP. Self-reported comorbidities, perceived needs, and sources for usual care for older and younger homeless adults. J Gen Intern Med 2005;20:726-30.

41. Adams J, Rosenheck R, Gee L, et al. Hospitalized younger: a comparison of a national sample of homeless and housed inpatient veterans. J Health Care Poor Underserved 2007;18:173-84.

42. Wright NM, Tompkins CN. How can health services effectively meet the health needs of homeless people? Br J Gen Pract 2006;56:286-93.

43. Hwang SW, Kirst MJ, Chiu S, et al. Multidimensional social support and the health of homeless individuals. J Urban Health 2009;86:791-803.

44. Bramley G, Fitzpatrick S, Edwards J, et al. Hard edges: mapping severe and multiple disadvantage in England. 2015. London: Lankelly Chase Foundation. https://lankellychase.org.uk/wp-content/uploads/ 2015/07/Hard-Edges-Mapping-SMD-2015.pdf (accessed 2 Mar 2017).

45. Wright NM, Tompkins CN. How can health services effectively meet the health needs of homeless people? $\mathrm{Br} J$ Gen Pract 2006;56:286-93.

46. Hwang SW, Tolomiczenko G, Kouyoumdjian FG, et al. Interventions to improve the health of the homeless: a systematic review. Am J Prev Med 2005;29:311.

47. Fitzpatrick-Lewis D, Ganann R, Krishnaratne S, et al. Effectiveness of interventions to improve the health and housing status of homeless people: a rapid systematic review. BMC Public Health 2011;11:638.

48. Collins SE, Malone DK, Clifasefi SL. Housing retention in single-site housing first for chronically homeless individuals with severe alcohol problems. Am J Public Health 2013;103 Suppl 2:S269-74.

49. Sadowski LS, Kee RA, VanderWeele TJ, et al. Effect of a housing and case management program on emergency department visits and hospitalizations among chronically ill homeless adults: a randomized trial. JAMA 2009;301:1771-8.

50. O'Toole TP, Johnson EE, Borgia ML, et al. Tailoring outreach efforts to increase primary care use among homeless veterans: results of a randomized controlled trial. J Gen Intern Med 2015;30:886-98. 
51. Hewett N, Buchman P, Musariri J, et al. Randomised controlled trial of GP-led in-hospital management of homeless people ('athway'). Clin Med 2016;16:223-9.

52. FEANTSA 2005: Federation of National Organisations working with the homeless. 2012. On the way home? FEANTSA monitoring report on homelessness and homeless policies in Europe. Brussels: FEANTSA. http://www.feantsa.org/en/report/2012/09/29/on-theway-home-feantsa-monitoring-report-on-homelessness-andhomelessness-policies-in-europe?bcParent=27 (accessed $24 \mathrm{Feb}$ 2017).

53. Effective Practice and Organisation of Care (EPOC). EPOC Resources for review authors. Oslo: Norwegian Knowledge Centre for the Health Services, 2015. http://epoc.cochrane.org/epocspecific-resources-review-authors (accessed Mar 2017).

54. Moher D, Liberati A, Tetzlaff J, et al. PRISMA Group. Preferred reporting items for systematic reviews and meta-analyses: the PRISMA statement. BMJ 2009;339:b2535.

55. The Cochrane Collaboration. In: Higgins JPT, Green S, eds. Cochrane Handbook for Systematic Reviews of Interventions Version 5.1.0. 2011. http://handbook.cochrane.org

56. Canavan R, Barry MM, Matanov A, et al. Service provision and barriers to care for homeless people with mental health problems across 14 European capital cities. BMC Health Serv Res 2012;12:222.
57. Gordon AJ, Montlack ML, Freyder P, et al. The Allegheny initiative for mental health integration for the homeless: integrating heterogeneous health services for homeless persons. Am J Public Health 2007:97:401-5.

58. NHS. Education for Scotland: a guide to good prescribing practice for prescribing pharmacists in NHS Scotland Jul 2012. www.nes. scot.nhs.uk. http://www.nes.scot.nhs.uk/education-and-training/bydiscipline/pharmacy/pharmacists/prescribing-and-clinical-skills.aspx (accessed Mar 2017).

59. Baird B, Charles A, Honeyman M, et al. Understanding pressures in general practice. Marylebone, England: The King's Fund, 2016.

60. Addicott R, Maguire D, Honeyman M, et al. Workforce planning in the NHS. Marylebone, England: The King's Fund, 2015.

61. Queen AB, Lowrie R, Richardson J, et al. Multimorbidity, disadvantage and patient engagement within a specialist homeless health service in the UK. BJGP Open. In Press;2017.

62. Coe AB, Moczygemba LR, Gatewood SBS, et al. Medication adherence challenges among patients experiencing homelessness in a behavioral health clinic. Res Soc Admin Pharm 2015;11:e110-20.

63. Milloy MJ, Kerr T, Bangsberg DR, et al. Homelessness as a structural barrier to effective antiretroviral therapy among HIV-seropositive illicit drug users in a Canadian setting. AIDS Patient Care STDS 2012;26:60-7. 